\title{
Some results on the qualitative properties of positive solutions of quasilinear elliptic equations
}

\author{
Berardino SCIUNZI* \\ Dipartimento di Matematica \\ Università di Roma "Tor Vergata" \\ Via della Ricerca Scientifica \\ 00133 Roma, Italy \\ e-mail: sciunzi@mat.uniroma2.it
}

\begin{abstract}
We consider the Dirichlet problem $-\Delta_{m}(u)=f(u)$ in $\Omega$ with zero Dirichlet boundary conditions. We prove local summability properties of $\frac{1}{|D u|}$ and we exploit these results to give geometric characterizations of the critical set $Z=\{x \in \Omega \mid D u(x)=0\}$. We extend to the case of changing sign nonlinearities some results known in the case $f(s)>0$ for $s>0$.

2000 Mathematics Subject Classification: 35B05,35B65,35J70.

Key words: $m$-Laplace operator, geometric and qualitative properties of the solutions, regularity of the solutions.
\end{abstract}

\section{Introduction and statement of the results}

Let us consider weak $C^{1}(\bar{\Omega})$ solutions of the problem

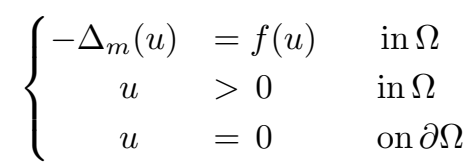

where $\Omega$ is a bounded smooth domain in $\mathbb{R}^{N}, N \geqslant 2, \Delta_{m}(u)=\operatorname{div}\left(|D u|^{m-2} D u\right)$ is the m-Laplace operator and $f: \mathbb{R} \rightarrow \mathbb{R}$ is a locally Lipschitz continuous function. Lineari'

*Supported by MURST, Project "Metodi Variazionali ed Equazioni Differenziali Non 
It is well known that, since the m-Laplace operator is singular or degenerate elliptic (respectively if $1<m<2$ or $m>2$ ) in the critical set

$$
Z \equiv\{x \in \Omega \mid D u(x)=0\}
$$

solutions of (1.1) belong generally to the class $C^{1, \tau}$ with $\tau<1$ (see $[6,13]$ ), and solve (1.1) only in the weak sense.

On the contrary the m-Laplace operator is a strictly elliptic operator in $\Omega \backslash Z$ and many standard results known in the literature can be successfully exploited there. As an example, solutions of (1.1) are smooth in $\Omega \backslash Z$.

Therefore the study of the critical set is very interesting and leads to many useful applications.

Nevertheless few results are known on this topic since the geometry of the critical set could be very irregular and many geometric approaches (as Hopf Lemma) do not provide satisfactory results.

In $[4,5]$ L. Damascelli and the author proved some results in the case when $f$ is positive. These results are based on summability properties of $\frac{1}{|D u|}$ which allow to get a weighted Sobolev type inequality in weighted Sobolev spaces. A weak Harnack type inequality for nonnegative solutions of the linearized equation then follows (see [5]) and provides a strong maximum principle for the linearized equations which is the key tool for the study of the critical set $Z$.

This paper deals with the case when the nonlinearity $f$ may change sign.

In this setting, as many examples show, global summability results for $\frac{1}{|D u|}$ like the ones in $[4,5]$ are not expected in general. In particular global summability results for $\frac{1}{|D u|}$ could follow for particular nonlinearities but there are no results in this direction.

In this paper we first prove a local version of the summability results for $\frac{1}{|D u|}$ which holds in regions where $f(u)$ is positive or in regions where $f(u)$ is negative (see Theorem 3.1).

Then, since the weighted Sobolev type inequality proved in [4] is local in nature, a weighted Sobolev type inequality follows in regions where $f(u)$ is positive or in regions where $f(u)$ is negative, and also allows to get weak Harnack type inequalities for nonnegative solutions of the linearized equation as in [5].

Therefore a strong maximum principle for the linearized operator follows, which is particulary interesting when applied to the derivatives of $u$ (see Corollary 4.1).

We will exploit these results together with some general geometric properties of the critical set proved in Section 5. In particular in Section 5 (see Proposition 5.1 ) we prove that there are not isolated points of $Z$ in regions where the nonlinearity $f$ is nondecreasing.

More precisely, we prove that, if $G \subset \Omega, f$ is nondecreasing in $u(G)$, and

$$
u_{x_{i}} \geqslant 0 \text { in } G \text { with } u_{x_{i}}>0 \text { on } \partial G
$$

then it follows that

$$
u_{x_{i}}>0 \text { in } G
$$

and consequently $G \cap Z=\emptyset$. 
This result is based only on the regularity results of the solutions proved in [4]. No assumptions on the sign of $f(u)$ are needed in this case.

This is a crucial point since it allows to exploit these results, which hold in regions where $f$ is nondecreasing, together with the strong maximum principle for the linearized operator (see Corollary 4.1), which holds in regions where $f$ is positive (or negative). As an application in Section 6 we assume that

$\mathrm{I}_{1}\{s \in \mathbb{R} \mid f(s)=0\} \equiv\left\{0, u_{0}\right\}\left(u_{0}>0\right)$.

$\mathrm{I}_{2} f^{\prime} \geqslant 0$ a.e. in $\left[u_{0}-\gamma ; u_{0}+\gamma\right]$ (for some $\gamma>0$ ).

so that $f$ is nondecreasing where it changes sign. With these assumptions we prove that, if the domain is a bounded convex and symmetric domain in $\mathbb{R}^{2}$ $\left(\frac{2 N+2}{N+2}<m \leqslant 2\right)$, then, assuming that 0 is the center of symmetry, we have

$$
Z \equiv\{x \in \Omega \mid D u(x)=0\}=\{0\}
$$

(Consequently $u \in C^{2}(\Omega \backslash\{0\})$ ).

As a model nonlinearity for this application we may consider

$$
f(s)=s^{q}-\Lambda s^{m-1} .
$$

For this kind of nonlinearity global summability results for $\frac{1}{|D u|}$ like the ones in $[4,5]$ are not known but could hold and could provide stronger results then the ones in this paper.

Anyway it is important to note that conditions $\mathrm{I}_{1}$ and $\mathrm{I}_{2}$ also allows to consider many other nonlinearities for which global summability results for $\frac{1}{|D u|}$ are not expected.

\section{Preliminaries}

In the sequel, as in [10], if $\rho \in L^{1}(\Omega)$, the space $H_{\rho}^{1, p}(\Omega)$ is defined as the completion of $C^{1}(\bar{\Omega})$ (or $\left.C^{\infty}(\bar{\Omega})\right)$ under the norm

$$
\|v\|_{H_{\rho}^{1, p}}=\|v\|_{L^{p}(\Omega)}+\|D v\|_{L^{p}(\Omega, \rho)}
$$

and $\|D v\|_{L^{p}(\Omega, \rho)}^{p}=\int_{\Omega}|D v|^{p} \rho d x$. In this way $H_{\rho}^{1, p}(\Omega)$ is a Banach space and $H_{\rho}^{1,2}(\Omega)$ is a Hilbert space. Moreover we define $H_{0, \rho}^{1, p}(\Omega)$ as the closure of $C_{c}^{1}(\bar{\Omega})$ $\left(\right.$ or $\left.C_{c}^{\infty}(\bar{\Omega})\right)$ in $H_{\rho}^{1, p}(\Omega)^{1}$.

From now on, given a fixed $C^{1}(\bar{\Omega})$ solution of $(1.1)$, we will consider

$$
\rho \equiv|D u|^{m-2}
$$

\footnotetext{
${ }^{1}$ We also recall that in [15] $H_{0, \rho}^{1, p}$ is defined as the space of functions having a distributional derivatives represented by a function for which the norm defined in (2.1) is bounded. These two definitions are equivalent if the domain has piecewise regular boundary (as is in our case).
} 
Therefore $\rho \in L^{\infty}(\Omega)$ if $m>2$ since $u \in C^{1}(\bar{\Omega})$. If instead $\frac{2 N+2}{N+2}<m<2$, then $\rho \in L^{1}(\Omega)$ as follows easily by the results of [4]. The point of view of considering $|D u|^{m-2}$ as a weight has been introduced in [1] where the case of radial solutions is considered and existence and uniqueness results are obtained.

Let us recall that the linearized operator at a fixed solution $u$ of (1.1), $L_{u}(v, \varphi)$, is well defined, for every $v, \varphi \in H_{\rho}^{1,2}(\Omega)$ (see Section 2 for details) with $\rho \equiv|D u|^{m-2}$, by

$$
\begin{aligned}
L_{u}(v, \varphi) \equiv & \int_{\Omega}\left[|D u|^{m-2}(D v, D \varphi)\right. \\
& \left.+(m-2)|D u|^{m-4}(D u, D v)(D u, D \varphi)-f^{\prime}(u) v \varphi\right] d x
\end{aligned}
$$

Moreover, $v \in H_{\rho}^{1,2}(\Omega)$ is a weak solution of the linearized equation if

$$
L_{u}(v, \varphi)=0
$$

for any $\varphi \in H_{0, \rho}^{1,2}(\Omega)$.

More generally, $v \in H_{\rho}^{1,2}(\Omega)$ is a weak supersolution (subsolution) of (2.3) if $L_{u}(v, \varphi) \geqslant 0(\leqslant 0)$ for any nonnegative $\varphi \in H_{0, \rho}^{1,2}(\Omega)$.

If $u$ is a $C^{1}(\bar{\Omega})$ solution of (1.1), then by [4] we have that

$$
|D u|^{m-2} D u \in W^{1,2}\left(\Omega, \mathbb{R}^{N}\right)
$$

and we can show that

$$
\begin{aligned}
L_{u}\left(u_{x_{i}}, \varphi\right) \equiv & \int_{\Omega}|D u|^{m-2}\left(D u_{x_{i}}, D \varphi\right) d x \\
& +\int_{\Omega}(m-2)|D u|^{m-4}\left(D u, D u_{x_{i}}\right)(D u, D \varphi)-f^{\prime}(u) u_{x_{i}} \varphi d x
\end{aligned}
$$

is well defined for every $1<m<\infty$ and for every $\varphi \in C_{0}^{1}(\Omega)$. Moreover the following equation holds

$$
L_{u}\left(u_{x_{i}}, \varphi\right)=0 \quad \forall \varphi \in C_{0}^{1}(\Omega) .
$$

Exploiting the linearized equation, in [4] some regularity results for the second derivatives of the solutions of (1.1) are proved. For the readers convenience we recall the statements here below.

Theorem 2.1 Let $u \in C^{1}(\Omega)$ be a weak solution of (1.1), with $f$ locally Lipschitz continuous $1<m<\infty$. Then, for any $E \subset \subset \Omega$ and for every $i, j=1, \ldots, N$, we have

$$
\sup _{x \in \Omega} \int_{E \backslash\left\{u_{x_{i}}=0\right\}} \frac{|D u|^{m-2}}{\left|u_{x_{i}}\right|^{\beta}|x-y|^{\gamma}}\left|D u_{i}\right|^{2} d y<C
$$


where $\beta<1, \gamma<N-2$ if $N \geqslant 3, \gamma=0$ if $N=2$ and $C=C(\beta, \gamma, E)$. Moreover

$$
\sup _{x \in \Omega} \int_{E \backslash Z} \frac{|D u|^{m-2-\beta}}{|x-y|^{\gamma}}\left\|D^{2} u\right\|^{2} d y<C,
$$

where $Z=\{x \in \Omega: D u(x)=0\}$ is the critical set of the solution.

Corollary 2.1 Let $u \in C^{1}(\Omega)$ be a weak solution of (1.1) with $f$ locally Lipschitz continuous, $1<m<\infty$. Then $u \in C^{2}(\Omega \backslash Z)$, where $Z=\{x \in \Omega: D u(x)=0\}$ is the critical set of the solution, $|D u|^{m-2} D u \in W_{l o c}^{1,2}\left(\Omega, \mathbb{R}^{N}\right)$, therefore $|D u|^{m-1} \in$ $W_{l o c}^{1,2}(\Omega)$.

If moreover $\Omega$ is smooth, $u \in C^{1}(\bar{\Omega})$ and $f$ is nonnegative and locally Lipschitz continuous in the closed interval $[0, \infty)$, then $Z \cap \partial \Omega=\emptyset, u \in C^{2}(\bar{\Omega} \backslash Z)$, $|D u|^{m-2} D u \in W^{1,2}\left(\Omega, \mathbb{R}^{N}\right)$ and $|D u|^{m-1} \in W^{1,2}(\Omega)$.

Remark 2.1 Let us remark that the fact that $u \in W^{2,2}(\Omega)$ if $1<m<2$ had been proved in the celebrated paper [12], among other regularity results for solutions of $(1.1)$.

Remark 2.2 We recall that under the assumptions at the boundary of Corollary 2.1 by [9] it follows that any solution $u$ of $(1.1)$ belongs to the class $C^{1, \tau}(\bar{\Omega})$. In particular in [9] G.M. Lieberman considers a more general class of operators with weaker assumptions on the solutions.

Remark 2.3 Since a $C^{1}(\Omega)$ solution $u$ of (1.1) with $f$ satisfying Locally Lipschitz continuous is regular in $\Omega \backslash Z$, the generalized derivatives of $|D u|^{m-2} u_{x_{i}}$ coincide there with the classical ones. Moreover in $\left\{u_{x_{i}}=0\right\}$, by Stampacchia's Theorem (see e.g. [14] Theorem 1.56 pp. 79), the generalized derivatives of $|D u|^{m-2} u_{x_{i}}$ are zero almost everywhere in $\left\{u_{x_{i}}=0\right\}$.

\section{Summability of the weight}

In this section we give a local version of summability properties of $\frac{1}{|D u|}$, in the spirit of the results in [4]. We recall that in [4] a positive nonlinearity $f$ is considered and global results are proved.

In particular we prove local summability properties of $\frac{1}{|D u|}$ in regions where $f(u)$ is positive (or negative). The proofs follow closely the one in [4] but some modifications are needed since we can not use the Hopf Lemma near the boundary of the regions considered which in our case may contain critical points of the solution.

Proposition 3.1 Let $\Omega$ be a domain in $\mathbb{R}^{N}$ and let $u \in C^{1}(\bar{\Omega})$ be a weak solution of (1.1) with $f$ locally Lipschitz continuous , $1<m<\infty$. 
Let $x^{0} \in \Omega$ and $\rho=\rho\left(x^{0}\right)>0$ be such that $f(u(x))>0$ in $B_{3 \rho}\left(x^{0}\right)$.

Then, we have that $\left|Z \cap B_{2 \rho}\left(x^{0}\right)\right|=0$, and for any $x \in B_{\rho}\left(x^{0}\right)$ and for every $r<1$, it follows

$$
\sup _{x \in B_{\rho}\left(x^{0}\right)} \int_{B_{2 \rho}\left(x^{0}\right)} \frac{1}{|D u|^{(m-1) r}} \frac{1}{|x-y|^{\gamma}} d y \leqslant C \sup _{B_{3 \rho}\left(x^{0}\right)}\left(\frac{1}{|f(u)|}\right)
$$

where $C$ depends on $\rho, \gamma<N-2$ if $N \geqslant 3$ and $\gamma=0$ if $N=2$.

Analogously, if $f(u(x))<0$ in $B_{3 \rho}\left(x^{0}\right)$ the same result follows.

Proof. We prove the result in the case when $f$ is positive in $B_{3 \rho}\left(x^{0}\right)$.

Given $x \in B_{\rho}\left(x^{0}\right)$, let us consider the cut-off function $\varphi_{\epsilon, x}(y) \in C_{0}^{\infty}\left(B_{3 \rho}\left(x^{0}\right)\right)$ such that

- $\operatorname{supp}\left(D \varphi_{\epsilon, x}\right) \subset\left\{B_{3 \rho}\left(x^{0}\right) \backslash B_{2 \rho}\left(x^{0}\right) \cup B_{2 \epsilon}(x) \backslash B_{\epsilon}(x)\right\}$.

- $\varphi_{\epsilon, x}=1$ in $B_{2 \rho}\left(x^{0}\right) \backslash B_{2 \epsilon}(x)$.

- $\varphi_{\epsilon, x}=0$ in $B_{\epsilon}(x)$

- $\left|D \varphi_{\epsilon, x}\right| \leq \frac{C}{\rho}$ in $B_{3 \rho}\left(x^{0}\right) \backslash B_{2 \rho}\left(x^{0}\right)$

- $\left|D \varphi_{\epsilon, x}\right| \leq \frac{C}{\epsilon}$ in $B_{2 \epsilon}(x) \backslash B_{\epsilon}(x)$

- Here above, $x$ is an arbitrary point in $B_{\rho}\left(x^{0}\right)$ and $\epsilon$ is a small parameter. For the moment we only assume that $\epsilon$ is small with respect to $\rho$. In particular, to make the above assumptions simultaneously fulfilled, we may and do assume that $B_{2 \epsilon}(x) \subset \subset B_{2 \rho}\left(x^{0}\right)$ since $x \in B_{\rho}\left(x^{0}\right)$.

Let us consider

$$
\psi_{\epsilon, x}(y)=\frac{1}{\left(|D u|^{m-1}+\epsilon\right)^{r}} \frac{1}{|x-y|^{\gamma}}
$$

with $\gamma$ and $r$ as in the statement of the proposition.

By the assumption on $\varphi_{\epsilon, x}$ it follows that

$$
\psi_{\epsilon, x} \varphi_{\epsilon, x}
$$

can be used as test function in (1.1), obtaining

$$
\begin{aligned}
\int_{\Omega} f(u)\left(\psi_{\epsilon, x} \varphi_{\epsilon, x}\right) d y= & \int_{\Omega}\left(|D u|^{m-2} D u, D\left(\psi_{\epsilon, x}\right) \varphi_{\epsilon, x} d y\right. \\
& +\int_{\Omega}\left(|D u|^{m-2} D u, D\left(\varphi_{\epsilon, x}\right)\left(\psi_{\epsilon, x}\right) d y\right.
\end{aligned}
$$


Consequently, exploiting the assumption that $f(u)>0$ in $B_{3 \rho}\left(x^{0}\right)$, we get

$$
\begin{aligned}
\int_{B_{3 \rho}\left(x^{0}\right)}\left(\psi_{\epsilon, x} \varphi_{\epsilon, x}\right) d y & =\int_{B_{3 \rho}\left(x^{0}\right)} \frac{f(u)}{f(u)}\left(\psi_{\epsilon, x} \varphi_{\epsilon, x}\right) d y \\
\leq & \sup _{B_{3 \rho}\left(x^{0}\right)}\left(\frac{1}{f(u)}\right) \int_{B_{3 \rho}\left(x^{0}\right)} f(u)\left(\psi_{\epsilon, x} \varphi_{\epsilon, x}\right) d y \\
\leq & \sup _{B_{3 \rho}\left(x^{0}\right)}\left(\frac{1}{f(u)}\right)\left\{\int_{B_{3 \rho}\left(x^{0}\right)}|D u|^{m-1}\left|D \psi_{\epsilon, x}\right| \varphi_{\epsilon, x} d y\right. \\
& \left.+\int_{B_{3 \rho}\left(x^{0}\right)}|D u|^{m-1}\left|D \varphi_{\epsilon, x}\right|\left(\psi_{\epsilon, x}\right) d y\right\}
\end{aligned}
$$

Let us now note that, since $\frac{|D u|^{m-1}}{\left(|D u|^{m-1}+\epsilon\right)^{r}} \leq|D u|^{(m-1)(1-r)} \leqslant$ const because $r<1$ and $u \in C^{1}(\Omega)$, then

$$
\begin{aligned}
\int_{B_{3 \rho}\left(x^{0}\right)}|D u|^{m-1}\left|D \varphi_{\epsilon, x}\right|\left(\psi_{\epsilon, x}\right) d y \leq & \int_{B_{3 \rho}\left(x^{0}\right)} \frac{\left|D \varphi_{\epsilon, x}\right|}{|x-y|^{\gamma}} d y \\
\leq & \int_{B_{3 \rho}\left(x^{0}\right) \backslash B_{2 \rho}\left(x^{0}\right)} \frac{\left|D \varphi_{\epsilon, x}\right|}{|x-y|^{\gamma}} d y \\
& +\int_{B_{2 \epsilon}(x) \backslash B_{\epsilon}(x)} \frac{\left|D \varphi_{\epsilon, x}\right|}{|x-y|^{\gamma}} d y \\
\leq & \text { const }+ \text { const } \frac{\epsilon^{N}}{\epsilon^{\gamma+1}} \leq \text { const }
\end{aligned}
$$

where we have used the fact that $\gamma+1<N$ and $r<1$ by assumption. Moreover $\frac{1}{|x-y|^{\gamma}} \leq$ const in $B_{3 \rho}\left(x^{0}\right) \backslash B_{2 \rho}\left(x^{0}\right)$ since $x$ is in $B_{\rho}\left(x^{0}\right)$. Also we recall that $\left|D \varphi_{\epsilon, x}\right| \leq \frac{C}{\epsilon}$ by assumption and we have $\frac{1}{|x-y|^{\gamma}} \leq \frac{C}{\epsilon^{\gamma}}$ in $B_{2 \epsilon}(x) \backslash B_{\epsilon}(x)$. Therefore,

$$
\begin{aligned}
& \int_{B_{3 \rho}\left(x^{0}\right)}\left(\psi_{\epsilon, x} \varphi_{\epsilon, x}\right) d y \\
& \quad \leq \text { const } \cdot \sup _{B_{3 \rho}\left(x^{0}\right)}\left(\frac{1}{f(u)}\right)\left\{1+\int_{B_{3 \rho}\left(x^{0}\right)}|D u|^{m-1}\left|D \psi_{\epsilon, x}\right| \varphi_{\epsilon, x} d y\right\}
\end{aligned}
$$

and

$$
\begin{aligned}
& \int_{B_{3 \rho}\left(x^{0}\right)}\left(\psi_{\epsilon, x} \varphi_{\epsilon, x}\right) d y \\
& \quad \leq \sup _{B_{3 \rho}\left(x^{0}\right)}\left(\frac{1}{f(u)}\right)\left\{\operatorname{const} \int_{B_{3 \rho}\left(x^{0}\right)} \frac{|D u|^{m-1}}{\left(|D u|^{m-1}+\epsilon\right)^{r+1}} \frac{|D u|^{m-2}\left\|D^{2} u\right\|}{|x-y|^{\gamma}} \varphi_{\epsilon, x} d y\right.
\end{aligned}
$$




$$
\begin{aligned}
& \left.+\operatorname{const} \int_{B_{3 \rho}\left(x^{0}\right)} \frac{|D u|^{m-1}}{\left(|D u|^{m-1}+\epsilon\right)^{r}} \frac{\varphi_{\epsilon, x}}{|x-y|^{\gamma+1}} d y+\text { const }\right\} \\
\leq & \sup _{B_{3 \rho}\left(x^{0}\right)} \frac{1}{f(u)}\left\{\text { const } \int_{B_{3 \rho}\left(x^{0}\right)} \frac{|D u|^{m-1}|D u|^{m-2}\left\|D^{2} u\right\| \varphi_{\epsilon, x}}{\left(|D u|^{m-1}+\epsilon\right)^{r+1}|x-y|^{\gamma}} d y+\text { const }\right\}
\end{aligned}
$$

Here above we used the fact that

$$
\int_{\Omega} \frac{|D u|^{m-1}}{\left(|D u|^{m-1}+\epsilon\right)^{r}} \frac{\varphi_{\epsilon, x}}{|x-y|^{\gamma+1}} d y \leq \mathrm{const} \int_{\Omega} \frac{1}{|x-y|^{\gamma+1}} d y \leq \mathrm{const}
$$

since $\frac{|D u|^{m-1}}{\left(|D u|^{m-1}+\epsilon\right)^{r}} \leq 1$ and $\gamma+1<N$ by assumption.

Now we end the proof arguing as in [4]. Therefore, for $\epsilon$ sufficiently small, we can write for any $\beta<1$

$$
\begin{aligned}
& \int_{B_{3 \rho}\left(x^{0}\right)} \frac{\varphi_{\epsilon, x}}{\left(|D u|^{m-1}+\epsilon\right)^{r}|x-y|^{\gamma}} d y \leqslant \sup _{B_{3 \rho}\left(x^{0}\right)}\left(\frac{1}{f(u)}\right)\{\text { const } \\
& \left.+ \text { const } \int_{B_{3 \rho}\left(x^{0}\right)} \frac{\left(\varphi_{\epsilon, x}\right)^{\frac{1}{2}}|D u|^{\frac{m-2-\beta}{2}}|| D^{2} u \| \mid}{|x-y|^{\frac{\gamma}{2}}} \frac{|D u|^{\frac{m-2+\beta}{2}}}{|D u|^{\frac{(m-1) r}{2}}} \frac{\left(\varphi_{\epsilon, x}\right)^{\frac{1}{2}}}{\left(|D u|^{m-1}+\epsilon\right)^{\frac{r}{2}}|x-y|^{\frac{\gamma}{2}}} d y\right\}
\end{aligned}
$$

For $\beta<1$ such that $r=\frac{m-2+\beta}{m-1}<1$, by Young's inequality $\left(a b \leqslant \sigma a^{2}+\right.$ $\left.b^{2} \backslash 4 \sigma\right)$, we can choose $\sigma$ small such that

$$
\begin{aligned}
& (1-\sigma) \int_{B_{3 \rho}\left(x^{0}\right)} \frac{\varphi_{\epsilon, x}}{\left(|D u|^{m-1}+\epsilon\right)^{r}} \frac{1}{|x-y|^{\gamma}} d y \\
& \quad \leqslant \sup _{B_{3 \rho}\left(x^{0}\right)}\left(\frac{1}{f(u)}\right)\left\{\text { const }+ \text { const } \int_{B_{3 \rho}\left(x^{0}\right)} \frac{|D u|^{m-2-\beta}\left\|D^{2} u\right\|^{2}}{|x-y|^{\gamma}} d y\right\} .
\end{aligned}
$$

Therefore, since by Theorem 2.1

$$
\int_{B_{3 \rho}\left(x^{0}\right)} \frac{|D u|^{m-2-\beta}\left\|D^{2} u\right\|^{2}}{|x-y|^{\gamma}} d y \leq \mathrm{const}
$$

it follows

$$
\int_{B_{3 \rho}\left(x^{0}\right)} \frac{\varphi_{\epsilon, x}}{\left(|D u|^{m-1}+\epsilon\right)^{r}} \frac{1}{|x-y|^{\gamma}} d y \leqslant C \cdot \sup _{B_{3 \rho}\left(x^{0}\right)}\left(\frac{1}{f(u)}\right)
$$

where $C$ does not depend on $x$.

Since $\frac{\varphi_{\epsilon, x}}{\left(|D u|^{m-1}+\epsilon\right)^{r}} \frac{1}{|x-y|^{\gamma}} \stackrel{\epsilon \rightarrow 0}{\rightarrow} \frac{1}{|D u|^{(m-1) r}} \frac{1}{|x-y|^{\gamma}}$ a.e. in $B_{2 \rho}\left(x^{0}\right) \backslash Z$, while it tends to $+\infty$ in $Z$, by Fatou's Lemma we get that $\left|Z \cap B_{2 \rho}\left(x^{0}\right)\right|=0$ and the thesis. 
We use now covering arguments to deduce an analogous of previous results in regions where $f(u)$ is positive (or negative). In particular we will prove a result which holds also in regions which are not smooth.

Theorem 3.1 Let $\Omega$ be a domain in $\mathbb{R}^{N}$ and $u \in C^{1}(\bar{\Omega})$ a weak solution of $(1.1)$ with $f$ locally Lipschitz continuous, $1<m<\infty$ and let $Z \equiv\{x \in \Omega \mid D u(x)=0\}$. Suppose $\Omega^{\prime} \subset \Omega$ and

$$
f(u(x)) \geqslant \theta>0 \quad \text { in } \quad \Omega^{\prime}
$$

or

$$
f(u(x)) \leqslant \theta^{\prime}<0 \quad \text { in } \Omega^{\prime}
$$

Then $\left|Z \cap \Omega^{\prime}\right|=0$ and, for any $x \in \Omega^{\prime}$ and for every $r<1, \gamma<N-2$ if $N \geqslant 3$ and $\gamma=0$ if $N=2$, we have

$$
\int_{\Omega^{\prime}} \frac{1}{|D u|^{(m-1) r}} \frac{1}{|x-y|^{\gamma}} d y \leqslant C
$$

where $C$ does not depend on $x$, but depends on $\Omega^{\prime}$.

Proof. We prove the result in the case

$$
f(u(x)) \geqslant \theta>0 \quad \text { in } \quad \Omega^{\prime}
$$

Consider in this case $\Omega^{\prime \prime}$ such that

$$
\Omega^{\prime} \subset \subset \Omega^{\prime \prime} \subset \subset\left\{x \in \Omega \mid f(u(x)) \geqslant \frac{\theta}{2}>0\right\}
$$

Let us note that given $p \in \Omega^{\prime}$, by Proposition 3.1, there exists a ball $B_{2 \rho_{p}}(p) \subset \Omega^{\prime \prime}$ such that, for any $x \in B_{\rho_{p}}(p)$, we have

$$
\int_{B_{2 \rho_{p}}(p)} \frac{1}{|D u|^{(m-1) r}} \frac{1}{|x-y|^{\gamma}} d y \leqslant{ }^{2} C\left(\rho_{p}, p\right)
$$

Consequently (3.10) and the fact that $\left|Z \cap \Omega^{\prime}\right|=0$ follows at once if $\gamma=0$ with simple covering arguments.

A little care is needed in the general case $\gamma<N-2$ because of the position of the pole $x$. We argue as follows.

\footnotetext{
${ }^{2}$ Here we mean that $C\left(\rho_{p}, p\right)$ depends on $\rho_{p}$ also via $\sup _{B_{3 \rho_{p}}(p)}\left(\frac{1}{f(u)}\right)$
} 
Since $\overline{\Omega^{\prime}}$ is compact, we may assume that

$$
\overline{\Omega^{\prime}} \subset \bigcup_{i=1}^{M} B_{\rho_{p_{i}}}\left(p_{i}\right)
$$

with $B_{2 \rho_{p_{i}}}\left(p_{i}\right)$ satisfying (3.13).

Given any $x \in \Omega^{\prime}$, we find $\bar{i}$ so that

$$
x \in B_{\rho_{p_{\bar{i}}}}\left(p_{\bar{i}}\right)
$$

Therefore, by (3.13), we get

$$
\begin{aligned}
\int_{\Omega^{\prime}} \frac{1}{|D u|^{(m-1) r}} \frac{1}{|x-y|^{\gamma}} d y \leqslant & \int_{B_{2 \rho_{p_{\bar{i}}}\left(p_{\bar{i}}\right)}} \frac{1}{|D u|^{(m-1) r}} \frac{1}{|x-y|^{\gamma}} d y \\
& +\int_{\Omega^{\prime} \backslash B_{2 \rho_{p_{\bar{i}}}\left(p_{\bar{i}}\right)}} \frac{1}{|D u|^{(m-1) r}} \frac{1}{|x-y|^{\gamma}} d y \\
\leqslant & \sup _{i=1, \ldots, N}\left\{C\left(\rho_{p_{i}}, p_{i}\right)\right\} \\
& +\left(\frac{1}{\min _{i=1, \ldots, N}\left\{\rho_{p_{i}}\right\}}\right)^{\gamma} \int_{\Omega^{\prime}} \frac{1}{|D u|^{(m-1) r}} d y \leq \mathrm{const}
\end{aligned}
$$

where we used that the result if true for $\gamma=0$ as remarked above, i.e.

$$
\int_{\Omega^{\prime}} \frac{1}{|D u|^{(m-1) r}} d y \leq \text { const }
$$

\section{Some consequences}

Once we have local summability properties of $\frac{1}{|D u|}$ then, by [4] a weighted Sobolev (and Poincaré) type inequality follows in weighted Sobolev spaces with weight $\rho=|D u|^{m-2}$. Also, as in [5], a weak Harnack type inequality for nonnegative solutions of the linearized equation follows.

For the readers convenience, we briefly recall the statements of these results referring to $[4,5]$ for the proofs.

Theorem 4.1 Let $u \in C^{1}(\bar{\Omega})$ be a weak solution of (1.1) with $f$ locally Lipschitz continuous. Define $\bar{p}^{*}$ by

$$
\frac{1}{\bar{p}^{*}}=\frac{1}{p}-\frac{1}{N}+\frac{2}{N p}\left(\frac{m-2}{m-1}\right)
$$

(consequently $\bar{p}^{*}>p$ for $m>2$ ). 
Suppose $\Omega^{\prime} \subset \Omega$ and

$$
f(u(x)) \geqslant \theta>0 \quad \text { in } \quad \Omega^{\prime}
$$

or

$$
f(u(x)) \leqslant \theta^{\prime}<0 \quad \text { in } \quad \Omega^{\prime}
$$

Then, for every $p \geqslant 2$ we get that there exists a positive constant $c_{0}=c_{0}(N, p, \rho, t$, $\gamma, \theta)$ such that the following weighted Sobolev's inequality holds

$$
\|v\|_{L^{p^{*}}\left(\Omega^{\prime}\right)} \leqslant c_{0}\|D v\|_{L^{p}\left(\Omega^{\prime}, \rho\right)},
$$

for any $v \in H_{0, \rho}^{1, p}\left(\Omega^{\prime}\right)^{3}$ and for any $p^{*}<\bar{p}^{*}$.

Moreover if $v \in H_{0, \rho}^{1, p}\left(\Omega^{\prime}\right)$ we have the following weighted Poincaré inequality

$$
\|v\|_{L^{p}\left(\Omega^{\prime}\right)} \leqslant C\left(\left|\Omega^{\prime}\right|\right)\|D v\|_{L^{p}\left(\Omega^{\prime}, \rho\right)}
$$

where $C(|\Omega|) \rightarrow 0$ if $|\Omega| \rightarrow 0$.

Theorem 4.2 Let $v \in H_{\rho}^{1,2}(\Omega) \cap L^{\infty}(\Omega)$ be a nonnegative weak supersolution of the linearized equation (2.3) in a bounded smooth domain $\Omega$ of $\mathbb{R}^{N}, N \geqslant 2$, with $f$ locally Lipschitz continuous and $m>2$. Suppose that $\overline{B(x, 5 \delta)} \subset \Omega$ and

$$
f(u(x)) \geqslant \theta>0 \quad \text { in } B(x, 5 \delta)
$$

or

$$
f(u(x)) \leqslant \theta^{\prime}<0 \quad \text { in } B(x, 5 \delta)
$$

Let us put

$$
\frac{1}{\overline{2}^{*}}=\frac{1}{2}-\frac{1}{N}+\frac{1}{N}\left(\frac{m-2}{m-1}\right)
$$

(consequently $\overline{2}^{*}>2$ for $m>2$ ) and let $2^{*}$ be any real number such that $2<2^{*}<$ $\overline{2}^{*}$. Then for every $0<s<\chi, \chi \equiv \frac{2^{*}}{2}$, there exists $C>0$ such that

$$
\|v\|_{L^{s}(B(x, 2 \delta))} \leqslant C \inf _{B(x, \delta)} v
$$

where $C$ is a constant depending on $x, s, N, u, m, f$.

If $\frac{2 N+2}{N+2}<m<2$ the same result holds with $\chi$ replaced by $\chi^{\prime} \equiv \frac{2^{\sharp}}{s^{\sharp}}$ where $2^{\sharp}$ is the classical Sobolev exponent, $\frac{2}{s^{\sharp}} \equiv 1-\frac{1}{s}$ and $s<\frac{m-1}{2-m}$.

${ }^{3}$ The proof of Theorem 4.1 (see [4]) is based on Theorem 3.1 and on potential estimates. Since potential estimates are also available for functions with zero mean (see [8]), then we can prove weighted Sobolev inequality, and weighted Poincaré inequality, also for functions with zero mean. 
Proof. It is sufficient to note that the proof of the Harnack inequality given in [5], is based on Sobolev weighted inequality which holds locally in our case as showed in Theorem 4.1

Corollary 4.1 Let $u \in C^{1}(\bar{\Omega})$ a weak solution of (1.1) with $m>2$ or $\frac{2 N+2}{N+2}<$ $m<2$ and $f$ locally Lipschitz continuous. Then, for any $i \in\{1, \ldots, N\}$ and any domain $\Omega^{\prime} \subset \Omega$ with

$$
f(u(x)) \geqslant \theta>0 \quad \text { in } \Omega^{\prime}
$$

or

$$
f(u(x)) \leqslant \theta^{\prime}<0 \quad \text { in } \Omega^{\prime}
$$

we have that, if $u_{x_{i}} \geqslant 0$ in $\Omega^{\prime}$, then either $u_{x_{i}} \equiv 0$ in $\Omega^{\prime}$ or $u_{x_{i}}>0$ in $\Omega^{\prime}$.

\section{The geometry of the critical set, via maximum principles}

In what follows the use of the linearized operator will be crucial. Let us recall that the linearized operator is defined by

$$
\begin{aligned}
L_{u}(v, \varphi) \equiv & \int_{\Omega}\left[|D u|^{m-2}(D v, D \varphi)\right. \\
& \left.+(m-2)|D u|^{m-4}(D u, D v)(D u, D \varphi)-f^{\prime}(u) v \varphi\right] d x
\end{aligned}
$$

In particular $L_{u}$ can be considered for $v, \varphi \in H_{\rho}^{1,2}$. Suppose moreover that $|D u|^{(m-2)} D v \in L^{2}\left(\Omega, \mathbb{R}^{N}\right)$ and that

$$
L_{u}(v, \varphi)=0 \quad \forall \varphi \in C_{c}^{\infty}(\Omega) .
$$

Then, by density arguments, it follows that

$$
L_{u}(v, \varphi)=0 \quad \forall \varphi \in H_{0}^{1,2}(\Omega) .
$$

We will now prove a general strong maximum principle for $L_{u}$. Next we will use it to deduce a geometric characterization of the critical set $Z$.

Theorem 5.1 Let $v \in H_{\rho}^{1,2}(\Omega) \cap C^{0}(\bar{\Omega})$ weakly solve

$$
L_{u}(v, \varphi)=0 \quad \forall \varphi \in H_{0, \rho}^{1,2}(\Omega) .
$$

Suppose that $f$ is a nondecreasing locally Lipschitz continuous, $\frac{2 N+2}{N+2}<m<\infty$. If $m>2$ assume also that ${ }^{4}|Z|=0$.

\footnotetext{
${ }^{4}$ This condition will be removed in the applications.
} 
If $G \subset \Omega$ is such that

$$
v \geqslant 0 \text { in } G, \text { and } v>0 \text { on } \partial G
$$

then $v>0$ in $G$.

The same result follows assuming only $f^{\prime}(u(x)) \geqslant 0$ for almost any $x \in G$.

Proof. Let $\gamma>0$ be such that $v \geqslant 2 \gamma$ on $\partial G$. Since $(v-\gamma)^{-} \in H_{0, \rho}^{1,2}(\Omega)$ it can be used as test function for the linearized equation, showing that

$$
\begin{gathered}
\int_{G}|D u|^{m-2}\left(D v, D(v-\gamma)^{-}\right) d x+\int_{G}(m-2)|D u|^{m-4}(D u, D v)\left(D u, D(v-\gamma)^{-}\right) d x \\
=\int_{G} f^{\prime}(u) v(v-\gamma)^{-} d x
\end{gathered}
$$

Since $f^{\prime}(u) \geqslant 0$ in $G$ and $v \geqslant 0$ in $G$, we get

$$
\begin{aligned}
& \int_{G}|D u|^{m-2}\left(D v, D(v-\gamma)^{-}\right) d x \\
& \quad+\int_{G}(m-2)|D u|^{m-4}(D u, D v)\left(D u, D(v-\gamma)^{-}\right) d x \geqslant 0
\end{aligned}
$$

i.e.

$$
\int_{G}|D u|^{m-2}\left|D(v-\gamma)^{-}\right|^{2}+(m-2)|D u|^{m-4}\left(D u, D(v-\gamma)^{-}\right)^{2} d x \leqslant 0
$$

To end the proof we now consider two cases (the case $\frac{2 N+2}{N+2}<m \leq 2$ and the case $2<m<\infty$ ).

Let us first assume $\frac{2 N+2}{N+2}<m \leq 2$. that

Since in this case $|D u|^{m-4}\left(D u, D(v-\gamma)^{-}\right)^{2} \leq|D u|^{m-2}\left|D(v-\gamma)^{-}\right|^{2}$ we have

$$
(m-2)|D u|^{m-4}\left(D u, D(v-\gamma)^{-}\right)^{2} \geq(m-2)|D u|^{m-2}\left|D(v-\gamma)^{-}\right|^{2}
$$

so that

$$
\begin{aligned}
\int_{G}(m-1)|D u|^{m-2}\left|D(v-\gamma)^{-}\right|^{2} d x & \leqslant \int_{G}|D u|^{m-2}\left|D(v-\gamma)^{-}\right|^{2} d x \\
& +\int_{G}(m-2)|D u|^{m-4}\left(D u, D(v-\gamma)^{-}\right)^{2} d x \\
& \leqslant 0
\end{aligned}
$$

Note now that $|D u|^{m-2}>0$ in $G$ in this case since $(m-2) \leq 0$ and $u \in$ $C^{1}(\bar{\Omega})$. Therefore

$$
\int_{G}\left|D(v-\gamma)^{-}\right|^{2} d x \leqslant 0
$$

which implies $\left|D(v-\gamma)^{-}\right|=0$ almost everywhere in $G$ and proves the thesis in this case. 
Let us now assume $2<m<\infty$.

Arguing as above we get

$$
\int_{G}|D u|^{m-2}\left|D(v-\gamma)^{-}\right|^{2}+(m-2)|D u|^{m-4}\left(D u, D(v-\gamma)^{-}\right)^{2} d x \leqslant 0
$$

and since in this case $(m-2)|D u|^{m-4}\left(D u, D(v-\gamma)^{-}\right)^{2} d x \geqslant 0$, we immediately get

$$
\int_{G}|D u|^{m-2}\left|D(v-\gamma)^{-}\right|^{2} d x \leqslant 0
$$

Having assumed that $Z=0$ if $m>2$, the thesis follows now as above, since $|D u|^{m-2}\left|D(v-\gamma)^{-}\right|^{2}=0$ a.e. in $G$ implies $\left|D(v-\gamma)^{-}\right|=0$ a.e. in $G$ and the thesis.

Our main purpose (see Proposition 5.1 here below) is to apply Theorem 5.1 to the case

$$
v=u_{x_{i}} \quad i=1, \ldots, N
$$

In this case we also avoid the a-priori assumption $|Z|=0$ needed in Theorem 5.1 for the case $m>2$ by slightly modifying the arguments in the proof.

Proposition 5.1 Let $u \in C^{1}(\bar{\Omega})$ be a weak solution of $(1.1), \frac{2 N+2}{N+2}<m<\infty$. Suppose that $f$ is locally Lipschitz continuous and suppose that $f^{\prime}(u(x)) \geqslant 0$ for any $x \in G$ where $G \subset \Omega$. Moreover assume that for some $1 \leqslant i \leqslant N u_{x_{i}} \geqslant 0$ in $G$ and $u_{x_{i}}>0$ on $\partial G$. Then $u_{x_{i}}>0$ in $G$ and, in particular

$$
G \cap Z \equiv \emptyset
$$

Proof. By Corollary 2.1 it follows that $u_{x_{i}} \in H_{\rho}^{1,2}(\Omega)$ and moreover $|D u|^{p-2}$ $\left|u_{x_{i} x_{j}}\right| \in L^{2}(\Omega)$. Moreover $L_{u}\left(u_{x_{i}}, \varphi\right)=0$ for all $\varphi \in C_{c}^{\infty}(\Omega)$ (see [4]). Therefore we can apply Theorem 5.1 and get the thesis for the case $\frac{2 N+2}{N+2}<m \leq 2$.

If else $m>2$ a little care is needed since here we are not making a-priori assumptions on the measure of $Z$ as in Theorem 5.1.

We argue as follows. Proceeding as in Theorem 5.1 with $v$ replaced by $u_{x_{i}}$, we get

$$
\int_{G}|D u|^{m-2}\left|D\left(u_{x_{i}}-\gamma\right)^{-}\right|^{2} d x \leqslant 0
$$

In a neighborhood of $\partial G$, there are not points where $D u=0$ because of the assumption $u_{x_{i}}>0$ on $\partial G$.

Suppose now by contradiction that $Z \cap G \neq \emptyset$. Then there exists a connected component $C$ of $G \backslash Z$ such that $\partial G \cap \bar{C} \neq \emptyset$ and $\bar{C} \cap Z \cap G \neq \emptyset$. get that

Therefore by (5.3), since $u_{x_{i}} \geq \gamma$ on the boundary of $G$ by assumption, we

$$
u_{x_{i}} \geqslant \gamma \quad \text { in } \quad C
$$

Consider now a point $p \in Z$ with $p \in \partial C$. 
At the same time we get $u_{x_{i}}(p) \geq \gamma>0$ by $(5.4)$, and $u_{x_{i}}(p)=0$ since $p \in Z$.

This contradiction shows that

$$
Z \cap G=\emptyset
$$

and the thesis.

The above result allows to prove immediately an important property of the critical set $Z$, as stated in the following

Corollary 5.1 Let $u \in C^{1}(\bar{\Omega})$ be a weak solution of (1.1). Suppose that $f$ is locally Lipschitz continuous.

Suppose that the domain $\Omega$ is convex and symmetric in the $e_{i}$-direction and the solution $u$ is symmetric and monotone, in the sense that $u_{x_{i}} \geqslant 0$ in $\Omega_{i}^{-}=\{x \in$ $\left.\Omega: x_{i}<0\right\}$.

Then, given $G_{i}^{-} \subset \Omega_{i}^{-}$with $u_{x_{i}}>0$ on $\partial G_{i}^{-}$and $f^{\prime}(u) \geqslant 0$ in $G_{i}^{-}$, it follows

$$
G_{i}^{-} \cap Z=\emptyset
$$

In particular, if $\Omega$ is symmetric with respect to all the variables and we assume that the center of symmetry is 0 , then the only isolated point of $Z$ is 0 .

Remark 5.1 Corollary (5.1) applies when symmetry and monotonicity properties of the solutions are known.

We refer to $[3,4]$ and the references therein for results on this topic.

\section{Applications}

The results proved above are interesting in itself, and may be also exploited to get stronger information on the geometry of the critical set of the solutions under stronger assumptions. In this section we will assume that $\Omega$ is a bounded smooth domain in $\mathbb{R}^{2}$ and:

$\mathrm{I}_{1}\{s \in \mathbb{R} \mid f(s)=0\} \equiv\left\{0, u_{0}\right\}\left(u_{0}>0\right)$.

$\mathrm{I}_{2} f^{\prime} \geqslant 0$ a.e. in $\left[u_{0}-\gamma ; u_{0}+\gamma\right]$ (for some $\gamma>0$ ).

Under these assumptions we extend to the case changing sign nonlinearities some results proved in [5] for the case of positive nonlinearities.

Let us fix some notations.

Let $\nu$ be a direction in $\mathbb{R}^{N}$. For a real number $\lambda$ we define

$$
\begin{aligned}
& T_{\lambda}^{\nu}=\{x \in \mathbb{R}: x \cdot \nu=\lambda\} \\
& \Omega_{\lambda}^{\nu}=\{x \in \Omega: x \cdot \nu<\lambda\}
\end{aligned}
$$


Theorem 6.1 Let $u \in C^{1}(\bar{\Omega})$ be a weak solution of (1.1).

Suppose that $\Omega$ is a bounded smooth domain in $\mathbb{R}^{2}$ which is strictly convex and symmetric in the $e_{i}$-direction for two orthogonal directions $e_{1}, e_{2}$ and assume that 0 is the center of symmetry ${ }^{5}$.

Assume $^{6} \frac{3}{2}<m \leqslant 2$ and $f$ locally Lipschitz continuous satisfying $\left(I_{1}\right)$ and $\left(I_{2}\right)$. Then

$$
Z \equiv\{x \in \Omega \mid D u(x)=0\}=\{0\}
$$

Consequently $u \in C^{2}(\Omega \backslash\{0\})$.

Proof. By Theorem 1.1 in [3] we have that $u$ is symmetric, monotone in the $e_{i}$-direction for $i=1,2$, and is strictly monotone outside the critical set $Z$.

We claim now that

$$
Z \subset U_{0} \cup\{0\}
$$

where $U_{0} \equiv\left\{x \in \Omega \mid u(x)=u_{0}\right\}$.

To prove this, let us assume the contrary and consider $x \in \Omega$ such that $x \neq 0$ and

$$
x \in Z \text { with } u(x) \neq u_{0}
$$

By continuity, it follows that there exists a ball $B_{\rho}(x)$ such that $f(u)$ is positive (or negative) in $B_{\rho}(x)$. Also by symmetry we may and do assume that $B_{\rho}(x) \subset \Omega_{0}^{e_{1}}$ and $u_{x_{1}} \geqslant 0$ in $B_{\rho}(x)$. Since $x \in Z$, then $u_{x_{1}}(x)=0$ and by Corollary 4.1 we gather $u_{x_{1}} \equiv 0$ in $B_{\rho}(x)$. Anyway the case $u_{x_{1}} \equiv 0$ in $B_{\rho}(x)$ is impossible since $u$ is strictly monotone outside $Z$ by [3] and $\left|B_{\rho}(x) \cap Z\right|=0$ by Theorem 3.1. This contradiction shows that it is not possible that $x \in Z$. Consequently (6.4) follows.

Assume now that the thesis of the Theorem is not true and that there exists a point $\left(x_{1}^{0}, x_{2}^{0}\right) \in Z$ with $\left(x_{1}^{0}, x_{2}^{0}\right) \neq 0$. In this case by $(6.4)$ we have $u\left(x_{1}^{0}, x_{2}^{0}\right)=u_{0}$.

Let us show that there exist $\epsilon^{\prime}$ and $\epsilon^{\prime \prime}$ such that

$$
D u\left(x_{1}, x_{2}^{0}+\epsilon^{\prime}\right) \neq 0 \quad \forall x_{1} \leqslant 0
$$

and

$$
D u\left(x_{1}, x_{2}^{0}-\epsilon^{\prime \prime}\right) \neq 0 \quad \forall x_{1} \leqslant 0
$$

To prove this we exploit a sliding balls technique introduced in [3] (see Proposition 3.1). It is crucial here a joint use of this technique and (6.4)(see figure 1).

\footnotetext{
${ }^{5}$ With the notations of [7] we are assuming $\lambda_{1}\left(e_{1}\right)=\lambda_{1}\left(-e_{1}\right)=\lambda_{1}\left(e_{2}\right)=\lambda_{1}\left(-e_{2}\right)=0$.

${ }^{6}$ Note that he condition $\frac{3}{2}<m<2$ is equivalent to the condition $\frac{2 N+2}{N+2}<m<2$ for $N=2$.
} 


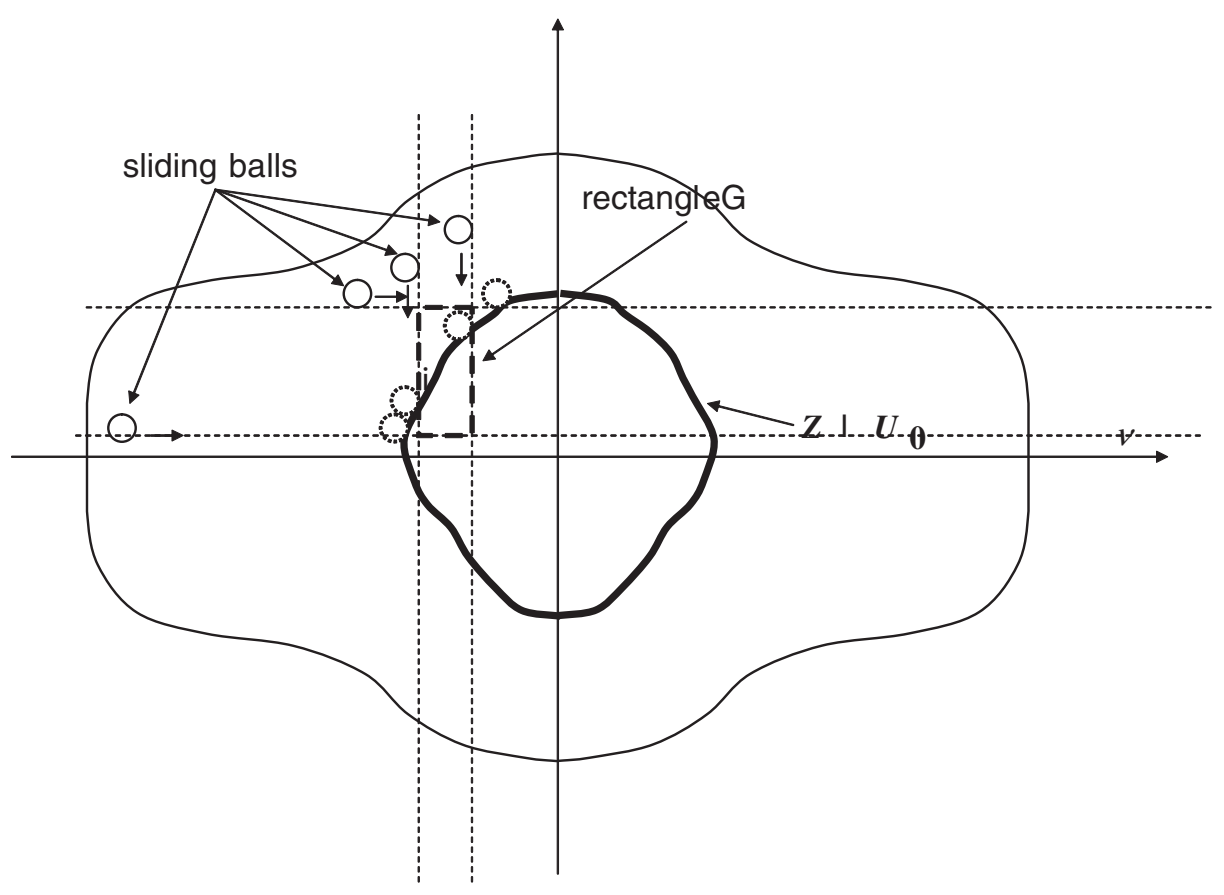

Figure 1 Construction of the rectangle $G$

Let us consider a ball $B_{\rho}(\xi)$ with $\xi_{2}>x_{2}^{0}+2 \rho$, and let us slide this ball from $-\infty$ to $\infty$ in the $e_{1}$-direction until it touches $U_{0}$ for the first time $t_{0}$ at a point $p$. More precisely we are assuming that

$$
\begin{array}{r}
B_{\rho}\left(\xi-t e_{1}\right) \cap U_{0}=\emptyset \text { for } t<t_{0} \\
B_{\rho}\left(\xi-t_{0} e_{1}\right) \cap U_{0} \ni p
\end{array}
$$

Note that, in what follows everything can be proved in a simpler way if $t_{0}$ does not exist.

Now we recall that $u$ is monotone nondecreasing in the $e_{1}$-direction in $\Omega_{0}^{e_{1}}$ by [3]. Therefore $u<u_{0}$ in the interior of $B_{\rho}\left(\xi-t_{0} e_{1}\right)$. We claim that this implies $D u(p) \neq 0$.

In fact, if we put $w \equiv\left(u-u_{0}\right)$, it follows that $-w \geq 0$ in $B_{\rho}\left(\xi-t_{0} e_{1}\right)$. Also we have that

$$
\begin{aligned}
& -\Delta_{m}(w)+\Lambda w=f(u)+\Lambda u-\Lambda u_{0}= \\
& =f(u)+\Lambda u-\left(f\left(u_{0}\right)+\Lambda u_{0}\right)
\end{aligned}
$$

recalling that $f\left(u_{0}\right)=0$, and we are assuming $\Lambda \geq 0$.

Now, since $f$ is locally Lipschitz continuous, we may and do assume that $\Lambda$ is sufficiently large so that $f(s)+\Lambda s$ is nondecreasing in $\left[0,\|u\|_{\infty}\right]$. With this 
assumption, since $w \leq 0\left(u \leq u_{0}\right)$ in $B_{\rho}\left(\xi-t_{0} e_{1}\right)$, it follows

$$
-\Delta_{m}(w)+\Lambda w \leq 0 \quad \text { in } \quad B_{\rho}\left(\xi-t_{0} e_{1}\right)
$$

i.e.

$$
-\Delta_{m}(-w)+\Lambda(-w) \geq 0 \quad \text { in } \quad B_{\rho}\left(\xi-t_{0} e_{1}\right)
$$

Therefore, by the strong maximum principle (see [16]), it follows

$$
D u(p) \neq 0
$$

To end the proof of (6.5) it is now sufficient to note that by monotonicity, the line parallel to $e_{1}$ through $p$ intersects the set $U_{0}$ in $\Omega_{0}^{e_{1}}$ only at $p$. More precisely, $U_{0} \cap \Omega_{0}^{e_{1}} \cap\left\{x \mid x_{2}=p_{2}\right\}=p$. Therefore by (6.4) we get (6.5) (and (6.6) in the same way).

Arguing in the same way and considering the $e_{2}$-direction we find $\theta^{\prime}$ and $\theta^{\prime \prime}$ such that

$$
D u\left(x_{1}^{0}+\theta^{\prime}, x_{2}\right) \neq 0 \quad \forall x_{2} \geqslant 0
$$

and

$$
D u\left(x_{1}^{0}-\theta^{\prime \prime}, x_{2}\right) \neq 0 \quad \forall x_{2} \geqslant 0
$$

Therefore we have that the rectangle $G$ of vertices $\left(x_{1}^{0}+\theta^{\prime}, x_{2}^{0}+\epsilon^{\prime}\right)\left(x_{1}^{0}+\theta^{\prime}, x_{2}^{0}-\epsilon^{\prime}\right)$ $\left(x_{1}^{0}-\theta^{\prime \prime}, x_{2}^{0}+\epsilon^{\prime}\right)\left(x_{1}^{0}-\theta^{\prime \prime}, x_{2}^{0}-\epsilon^{\prime \prime}\right)$ has no points of $Z$ on its boundary.

In particular we can suppose that $G$ is sufficiently small in order that $\mid u(x)-$ $u_{0} \mid \leqslant \frac{\gamma}{2}$ so that $f^{\prime}(u(x)) \geqslant \theta>0$ in $G$ (see figure 1 ).

If we assume now $\left(x_{1}^{0}, x_{2}^{0}\right) \neq(0,0)$ by symmetry we can suppose $G \subset \Omega_{0}^{e_{1}}$ and by construction

$$
u_{x_{1}}>0 \text { on } \partial G \text {. }
$$

Also we can assume $u_{x_{1}} \geqslant 0$ in $G$ (or $G \subset \Omega_{\lambda_{2}\left(e_{2}\right)}^{e_{2}}$ and $u_{x_{2}} \geqslant 0$ in $G$ ). In both cases we can apply Proposition 5.1 and get

$$
Z \cap G=\emptyset
$$

proving that

$$
Z \equiv\{0\}
$$

\section{Acknowledgement}

I would like to thank Prof. Lucio Damascelli for many useful comments and conversations related to this paper. 


\section{References}

[1] A. AFTALION and F. PACELLA, Morse index and uniqueness for positive radial solutions of $p$-Laplace equations, Trans. Amer. Mat. soc., 356(11) (2004), 4255-4272.

[2] L. DAMASCELLI, Comparison theorems for some quasilinear degenerate elliptic operators and applications to symmetry and monotonicity results, Ann. Inst. H. Poincaré. Analyse non linéaire, 15(4) (1998), 493-516.

[3] L. DAMASCELLI and F. PACELLA, Monotonicity and symmetry of solutions of p-Laplace equations, $1<p<2$, via the moving plane method. Ann. Scuola Norm. Sup. Pisa Cl. Sci., 26(4) (1998), 689-707.

[4] L. DAMASCELLI and B. SCIUNZI, Regularity, Monotonicity and Symmetry of positive solutions of $m$-Laplace equations. J. Diff. Equations. 206(2) (2004), 483-515.

[5] L. DAMASCELLI and B. SCIUNZI, Harnack Inequalities, Maximum and Comparison Principles, and Regularity of positive solutions of $m$-Laplace equations. Calculus of Variations and P.D.E. 25(2) (2006), 139-159.

[6] E. DIBENEDETTO, $C^{1+\alpha}$ local regularity of weak solutions of degenerate elliptic equations, Nonlinear Anal., 7(8) (1983), 827-850.

[7] B. GIDAS, W. M. NI and L. NIRENBERG, Symmetry and related properties via the maximum principle, Comm. Math. Phys., (68) (1979), 209-243.

[8] D. GILBARG and N. S. TRUDINGER, Elliptic partial differential equations of second order, Springer, second edition, 1983.

[9] G. M. LIEBERMAN, Boundary regularity for solutions of degenerate elliptic equations, Nonlinear Anal., 12(11) (1988), 1203-1219.

[10] M. K. V. MURTHY and G. STAMPACCHIA, Boundary value problems for some degenerate-elliptic operators, Ann. Mat. Pura Appl. 80(4) (1968), $1-122$.

[11] J. SERRIN and H. ZOU, Symmetry of Ground states of quasilinear elliptic equations, Arch. Rational Mech. Anal., 148 (1999), 265-290.

[12] J. SERRIN and H. ZOU, Cauchy-Liouville and universal boundedness theorems for quasilinear elliptic equations and inequalities, Acta Mathematica, 189 (2002).

[13] P. TOLKSDORF, Regularity for a more general class of quasilinear elliptic equations, J. Diff. Equations, (51) (1984), 126-150. 
[14] G. M. TROIANIELLO, Elliptic differential equations and obstacle problems, Plenum 1987.

[15] N. S. TRUDINGER, Linear elliptic operators with measurable coefficients, Ann. Scuola Norm. Sup. Pisa., 27(3) (1973), 265-308

[16] J. L. VAZQUEZ, A strong maximum principle for some quasilinear elliptic equations, Appl. Math. Optim., (1984), 191-202.

Received and accepted 6 June 2005

Published Online First 21 September 2007

To access this journal online:

http://www.birkhauser.ch 